\title{
Responder Threshold for Patient-Oriented Eczema Measure (POEM) and Children's Dermatology Life Quality Index (CDLQI) in Adolescents with Atopic Dermatitis
}

\author{
Eric L. Simpson - Marjolein de Bruin-Weller - Laurent Eckert • \\ Diane Whalley · Isabelle Guillemin · Matthew Reaney • \\ Zhen Chen · Lauren Nelson · Shanshan Qin · Ashish Bansal • \\ Abhijit Gadkari
}

Received: August 15, 2019 / Published online: October 22, 2019

(C) The Author(s) 2019

\begin{abstract}
Introduction: The Patient-Oriented Eczema Measure (POEM) assesses patient-reported frequency of atopic dermatitis (AD) symptoms, while the Children's Dermatology Life Quality Index (CDLQI) measures the impact of skin disease on health-related quality of life (HRQoL) in children. There is currently no threshold for clinically meaningful within-person change in POEM or CDLQI scores in adolescents. Here we
\end{abstract}

Enhanced Digital Features To view enhanced digital features for this article go to https://doi.org/10.6084/ m9.figshare.9929048.

Electronic supplementary material The online version of this article (https://doi.org/10.1007/s13555019-00333-2) contains supplementary material, which is available to authorized users.

E. L. Simpson $(\bowtie)$

Department of Dermatology, Oregon Health \&

Science University, Portland, OR, USA

e-mail: simpsone@ohsu.edu

M. de Bruin-Weller

University Medical Center Utrecht, Utrecht, The

Netherlands

L. Eckert

Sanofi, Chilly-Mazarin, France

D. Whalley

RTI Health Solutions, Manchester, UK empirically derive within-person thresholds of meaningful within-person change in POEM and CDLQI scores in adolescents with moderate-tosevere AD.

Methods: Data were used from a phase 3, randomized, double-blind, placebo-controlled trial of dupilumab in adolescents (aged $\geq 12$ to $<18$ years) with moderate-to-severe AD. Anchor-based methods were employed using the mean change in POEM and CDLQI scores from baseline to week 16 linked with a 1-point improvement in Patient Global Assessment of Disease (PGAD), a score of "a little better" on the Patient Global Assessment of Treatment effect (PGAT), a 50-74\% improvement from baseline in the Eczema Area and Severity Index (EASI50-74), and a 1-point improvement in Investigator's Global Assessment (IGA) score.

Results: A mean change of -7.8 and -5.6 in the POEM score was associated with PGAD and

I. Guillemin

Sanofi, Lyon, France

M. Reaney

Sanofi, Guildford, UK

Z. Chen · A. Bansal · A. Gadkari

Regeneron Pharmaceuticals, Inc., Tarrytown, NY, USA

L. Nelson · S. Qin

RTI Health Solutions, Durham, NC, USA 
PGAT anchors, respectively. EASI-50-74 was associated with a mean change in POEM score of -8.2 , while the IGA anchor was associated with a mean change of -7.9 in POEM score. The mean changes in CDLQI score associated with PGAD and PGAT anchors were -6.4 and - 6.6, respectively, while CDLQI mean scores changed by -8.3 and -8.0 for the EASI and IGA anchors, respectively.

Conclusion: In adolescents (aged $\geq 12$ to $<18$ years) with moderate-to-severe $\mathrm{AD}$, a within-person change of 6-8 points in POEM and CDLQI scores, independently, can be considered a reasonable responder threshold for clinically meaningful change in each of the two scales, respectively.

Trial Registration: ClinicalTrials.gov Identifier: NCT03054428.

Funding: Sanofi and Regeneron Pharmaceuticals, Inc.

Keywords: Adolescents; Atopic dermatitis; CDLQI; Dupilumab; POEM; Quality of life; Responder threshold; Validation

\section{INTRODUCTION}

It is important to understand patient-reported symptoms and impact of atopic dermatitis (AD) to comprehend the burden of living with $\mathrm{AD}$ and interpret purported treatment benefits. These burdens and benefits may be different in adolescents with $\mathrm{AD}$ compared with adults with $\mathrm{AD}$.

The Patient-Oriented Eczema Measure (POEM) assesses patient-reported frequency of AD symptoms in adults and children [1]. Gerbens et al. evaluated the measurement properties of the POEM and recommended the use of the tool in clinical trials [2]. Furthermore, the Harmonising Outcome Measures for Eczema (HOME) initiative also recommended the POEM as the core outcome instrument for assessing patient-reported $\mathrm{AD}$ symptoms in clinical trials [3]. The Dermatology Life Quality Index (DLQI) is frequently used to assess dermatology-specific health-related quality of life (HRQoL) in adult patients [4] but is not appropriate for pediatric patients. A version for pediatric patients, the
Children's Dermatology Life Quality Index (CDLQI), has been developed for patients aged $3-16$ years [5]. The CDLQI is a well-established and widely used tool that was used in 102 studies across 14 different skin conditions between 1995 and 2012 [6].

For adults with $\mathrm{AD}$, a within-person responder threshold for clinically meaningful change of four points has been established for both POEM [7] and DLQI [8]. However, currently, there is no validated within-person responder threshold for clinically meaningful within-person change in POEM or CDLQI scores in adolescents. The objective of this study was to empirically derive within-person responder thresholds of clinically meaningful change in POEM and CDLQI scores, independently, in adolescents with moderate-to-severe AD. ${ }^{1}$

\section{METHODS}

This analysis is based on data from a randomized, double-blind, placebo-controlled trial of dupilumab in adolescents with moderate-tosevere AD. Detailed descriptions of the study population, methodology, and efficacy and safety were previously reported [9]. The LIBERTY AD ADOL phase 3 trial included adolescent patients (aged 12 to $<18$ years) with moderate-to-severe AD inadequately controlled with topical therapies, who were randomized (1:1:1) to dupilumab every 2 weeks $(200 \mathrm{mg}$ if baseline body weight $<60 \mathrm{~kg}, 300 \mathrm{mg}$ if $\geq 60 \mathrm{~kg}$ ), $300 \mathrm{mg}$ dupilumab every 4 weeks , or placebo, for 16 weeks. The POEM and CDLQI were administered to patients in this trial at screening, baseline, and at weeks $2,4,8,12$, and 16. Of the 251 patients randomized in the LIBERTY AD ADOL trial, 250 (99.6\%) received $\geq 1$ dose of dupilumab or placebo and had $\geq 1$ postbaseline POEM or CDLQI assessment during the treatment period and were therefore included in the present analysis.

\footnotetext{
$\overline{1}$ Since only a within-person response is being considered, the term "minimal clinically important difference" (MCID) is not used, as the MCID can define a clinically meaningful difference for either a within-patient change or a cross-population mean change in score.
} 


\section{Instruments}

The POEM [1] is a 7-item questionnaire inquiring about AD-specific symptoms in the preceding week; each item is scored $0-4$, for a total score of $0-28$. A decrease in POEM score indicates a reduction (improvement) in the frequency of symptoms of AD. The CDLQI [5] is a 10-item questionnaire measuring the effect of skin disease on patient HRQoL in the preceding week. Each CDLQI item is scored 0-3, for a total score of 0-30; lower scores indicate lesser impairment in HRQoL.

\section{Estimation of Within-Person Change}

Anchor-based approaches were primarily used to define responder thresholds per the patientreported outcomes guidance from the US Food and Drug Administration [10], with distribution-based methods used as a secondary approach.

For anchor-based methods, the mean change in POEM score and, separately, the mean change in CDLQI score from baseline to week 16 were calculated for adolescents with a defined improvement in two patient-reported outcome (PRO) anchors, namely, a 1-point improvement in the Patient Global Assessment of Disease (PGAD) score and a score of "a little better" on the Patient Global Assessment of Treatment effect (PGAT), and in two clinicianreported outcome (ClinRO) anchors, namely, a 50-74\% improvement from baseline in the Eczema Area and Severity Index (EASI) (EASI50-74), and a 1-point improvement in the Investigator's Global Assessment (IGA) score.

Distribution-based methods included onehalf of standard deviation (SD) at baseline and the standard error of measurement (SEM) at baseline (assessed as SEM $=\mathrm{SD} \sqrt{1-r}$, where $r$ is the test-retest reliability). All analyses were performed using SAS software version 9.4 (SAS Institute Inc.; Cary, NC, USA).

The study was conducted in accordance with the provisions of the Declaration of Helsinki 1964, as revised in 2013, the International Council on Harmonization Good Clinical Practice (ICH GCP) guideline, and applicable regulatory requirements; the protocol was reviewed and approved by institutional review boards/ethics committees at all study sites (Electronic Supplementary Material Table). For all patients, written informed consent was obtained from a parent or legal guardian, and written informed assent was obtained from the patient.

\section{RESULTS}

The within-person responder thresholds for clinically meaningful change for POEM and CDLQI are shown in Table 1.

\section{POEM}

For the PRO anchors, a mean change of -7.8 and -5.6 in the POEM score was associated with improvement in the PGAD and PGAT, respectively. For the ClinRO anchors, a mean change of -8.2 and -7.9 in the POEM score was associated with improvement in EASI and IGA, respectively.

The distribution-based one-half of SD at baseline was 2.6 and the SEM was 2.3.

\section{CDLQI}

For the PRO anchors, a mean change of -6.4 and -6.6 in the CDLQI score was associated with improvement in the PGAD and PGAT, respectively. For the ClinRO anchors, a mean change of -8.3 and -8.0 in the CDLQI score was associated with improvement in the EASI and IGA, respectively.

The distribution-based one-half of SD at baseline was 3.4 and the SEM was 2.8 .

\section{DISCUSSION}

To the best of our knowledge, there are no previous reports that clearly define a responder threshold for POEM and CDLQI in this target population of adolescents with moderate-tosevere $\mathrm{AD}$. 
Table 1 Estimated thresholds for clinically meaningful change for the Patient-Oriented Eczema Measure and Children's Dermatology Life Quality Index scales in adolescents with moderate-to-severe atopic dermatitis

\begin{tabular}{|c|c|c|c|c|}
\hline \multirow[t]{2}{*}{ Estimation methods } & \multicolumn{2}{|l|}{ POEM } & \multicolumn{2}{|l|}{ CDLQI } \\
\hline & $\begin{array}{l}\text { Mean change from } \\
\text { baseline to Week } 16\end{array}$ & $\begin{array}{l}\text { Median change from } \\
\text { baseline to Week } 16\end{array}$ & $\begin{array}{l}\text { Mean change from } \\
\text { baseline to Week } 16\end{array}$ & $\begin{array}{l}\text { Median change from } \\
\text { baseline to Week } 16\end{array}$ \\
\hline \multicolumn{5}{|l|}{ Anchor-based estimates } \\
\hline $\begin{array}{l}\text { PGAD improvement of } \\
1 \text { point }(n=95)\end{array}$ & -7.8 & -8.0 & -6.4 & -6.0 \\
\hline $\begin{array}{l}\text { PGAT score of "a little } \\
\text { better" }(n=75)\end{array}$ & -5.6 & -4.0 & -6.6 & -6.0 \\
\hline EASI-50-74 $(n=55)$ & -8.2 & -8.0 & -8.3 & -8.0 \\
\hline $\begin{array}{l}\text { IGA improvement of } 1 \\
\text { point }(n=92)\end{array}$ & -7.9 & -8.0 & -8.0 & -8.0 \\
\hline \multicolumn{5}{|l|}{ Distribution-based estimates } \\
\hline $\begin{array}{l}\text { One-half of SD at } \\
\text { baseline }\end{array}$ & 2.6 & & 3.4 & \\
\hline SEM & 2.3 & & 2.8 & \\
\hline
\end{tabular}

CDLQI Children's Dermatology Life Quality Index, EASI-50-74 50-74\% improvement from baseline in Eczema Area and Severity Index, IGA Investigator's Global Assessment, PGAD Patient Global Assessment of Disease, PGAT Patient Global Assessment of Treatment, POEM Patient-Oriented Eczema Measure, $S D$ standard deviation, SEM standard error of the mean

Using anchor-based methods in adults, Schram et al. estimated a within-person responder definition for POEM as 3.4 [7]. This equates to a change in POEM score of 4 points in an individual patient as POEM is scored in integers. The COMET randomized feasibility study estimated the mean within-person change threshold for POEM as 4.27 using parent-reported data of children with $\mathrm{AD}$ aged between 1 month and 5 years to derive a responder threshold [11]. The estimated threshold from receiver operating characteristic (ROC) curve analysis was 3, whereas the distribution-based estimates were 2.5 (effect size) and 2.9 (one-half of SD). More recently, Howells et al. evaluated data from the randomized, controlled CLOTHES trial that included 300 children aged $1-15$ years (mean age 5 years) with moderate-to-severe AD [12]. A change of 1 point on a 6-point Patient/Parent Global Assessment (P/PGA) or on a 6-point IGA were used as predefined anchors of clinically relevant change, from which the mean within-person meaningful change in POEM was 6.13 and 5.25, respectively. Other anchor-based estimates of meaningful change using the P/PGA and IGA were 4.75 and 5.38 based on ROC curve analysis, and 4.52 and 4.43 based on logistic regression models, respectively. The distributionbased one-half of SD was 2.68. Howells et al. [12] combined the findings from other studies along with their own results and recommended that changes in POEM of $<3$ points were unlikely to be clinically important, changes of 3.0-3.9 points were probably clinically important, and changes of $\geq 4$ points were very likely to be clinically important. Distribution-based estimates based on our trial data (2.6 and 2.3) were considerably lower than the anchor-based estimates, but similar to estimates provided in the literature $[11,12]$. Although the CLOTHES trial also included adolescent patients [12], the responder definition is not provided separately for adolescents and the results do not directly 
apply to a population of adolescents (12 to $<18$ years) with moderate-to-severe AD.

No previous publications have estimated a meaningful within-person change threshold for the CDLQI score in adolescent patients with moderate-to-severe AD. Our results presented here suggest that a reasonable clinically meaningful change in CDLQI would range between 6.4 and 8.3 points, equivalent to a within-person score change of 6-8 points, given that CDLQI is only scored in integers.

The distribution estimates of 3.4 and 2.8 are comparable with the one-half of-SD of 2.8 based on published CDLQI scores in AD [5] but, similar to POEM results, are considerably lower than the anchor-based estimates derived from our trial data. Further work is warranted to estimate an appropriate within-person responder definition in children aged $<12$.

A limitation of this analysis is that the EASI score, although containing a component measuring lesions on the head region, is not correlated with quality of life assessed by CDLQI. However, we have mitigated this limitation by using other instruments, such as PGAD and PGAT, in our anchor-based methods for determining appropriate within-person responder definitions.

\section{CONCLUSION}

In adolescents with moderate-to-severe $\mathrm{AD}$, a within-person change of 6-8 points in the POEM and CDLQI scores, independently, can be considered to be a reasonable responder threshold for clinically meaningful change in each of the two scales, respectively.

\section{ACKNOWLEDGEMENTS}

The authors thank Ana B. Rossi and Paola MinaOsorio for their contributions, and the patients and their caregivers/families for participating in the study.

Funding. The study was sponsored by Sanofi and Regeneron Pharmaceuticals, Inc. The journal Rapid Service Fee was sponsored by Sanofi Genzyme and Regeneron Pharmaceuticals, Inc. All authors had full access to all of the data in this study and take complete responsibility for the integrity of the data and accuracy of the data analysis.

Medical Writing and Editorial Assistance. Medical writing/editorial assistance provided by Juliet Bell, PhD, and Grace Richmond, $\mathrm{PhD}$, of Excerpta Medica, funded by Sanofi Genzyme and Regeneron Pharmaceuticals, Inc.

Authorship. All named authors meet the International Committee of Medical Journal Editors (ICMJE) criteria for authorship for this article, take responsibility for the integrity of the work as a whole, and have given their approval for this version to be published.

Prior Presentation. Data were previously presented at the Revolutionizing Atopic Dermatitis conference (RAD 2019); Chicago, IL, USA; April 6-7, 2019.

Disclosures. Eric L. Simpson has been an investigator for AbbVie, Eli Lilly, Galderma, Kyowa Hakko Kirin, LEO Pharma, Merck, Pfizer, Regeneron Pharmaceuticals, Inc.; has received consultant honoraria from AbbVie, Boehringer Ingelheim, Dermavant, Eli Lilly, Forte Bio, Incyte, LEO Pharma, Menlo Therapeutics, Pfizer, Pierre Fabre Dermo Cosmétique, Regeneron Pharmaceuticals, Inc., Sanofi Genzyme, Valeant. Marjolein de Bruin-Weller has been Principal Investigator, advisory board member, consultant for Regeneron Pharmaceuticals, Inc., Sanofi Genzyme; Principal Investigator, advisory board member for AbbVie, Pfizer; advisory board member for Eli Lilly, UCB. Laurent Eckert is an employee of Sanofi, may hold stock and/or stock options in Sanofi. Isabelle Guillemin is an employee of Sanofi, may hold stock and/or stock options in Sanofi. Matthew Reaney is an employee of Sanofi, may hold stock and/or stock options in Sanofi. Diane Whalley is an employee of RTI Health Solutions, which received funding from Regeneron Pharmaceuticals, Inc. Lauren Nelson is an employee of RTI Health Solutions, which received funding from 
Regeneron Pharmaceuticals, Inc. Shanshan Qin is an employee of RTI Health Solutions, which received funding from Regeneron Pharmaceuticals, Inc. Zhen Chen is an employee and shareholder of Regeneron Pharmaceuticals, Inc. Ashish Bansal is an employee and shareholder of Regeneron Pharmaceuticals, Inc. Abhijit Gadkari is an employee and shareholder of Regeneron Pharmaceuticals, Inc.

Compliance with Ethics Guidelines. The study was conducted in accordance with the provisions of the Declaration of Helsinki 1964, as revised in 2013, the ICH GCP guideline, and applicable regulatory requirements; the protocol was reviewed and approved by institutional review boards/ethics committees at all study sites (Electronic Supplementary Material Table). For all patients, written informed consent was obtained from a parent or legal guardian, and written informed assent was obtained from the patient.

Data Availability. Qualified researchers may request access to study documents (including the clinical study report, study protocol with any amendments, blank case report form, statistical analysis plan) that support the methods and findings reported in this manuscript. Individual anonymized participant data will be considered for sharing once the indication has been approved by a regulatory body, if there is legal authority to share the data and there is not a reasonable likelihood of participant re-identification. Submit requests to https://errs.regeneron.com/external.

Open Access. This article is distributed under the terms of the Creative Commons Attribution-NonCommercial 4.0 International License (http://creativecommons.org/licenses/ by-nc/4.0/), which permits any noncommercial use, distribution, and reproduction in any medium, provided you give appropriate credit to the original author(s) and the source, provide a link to the Creative Commons license, and indicate if changes were made.

\section{REFERENCES}

1. Charman CR, Venn AJ, Williams HC. The patientoriented eczema measure: development and initial validation of a new tool for measuring atopic eczema severity from the patients' perspective. Arch Dermatol. 2004;140(12):1513-9.

2. Gerbens LAA, Prinsen CAC, Charmers JR, et al. Evaluation of the measurement properties of symptom measurement instruments for atopic eczema: a systematic review. Allergy. 2017;72(1):146-63.

3. Chalmers JR, Simpson E, Apfelbacher CJ, et al. Report from the fourth international consensus meeting to harmonize core outcome measures for atopic eczema/dermatitis clinical trials (HOME initiative). Br J Dermatol. 2016;175(1):69-79.

4. Finlay AY, Khan GK. Dermatology Life Quality Index (DLQI) - a simple practical measure for routine clinical use. Clin Exp Dermatol. 1994;19(3):210-6.

5. Lewis-Jones MS, Finlay AY. The Children's Dermatology Life Quality Index (CDLQI): initial validation and practical use. $\mathrm{Br} \mathrm{J}$ Dermatol. 1995;132(6):942-9.

6. Salek MS, Jung S, Brincat-Ruffini LA, et al. Clinical experience and psychometric properties of the children's dermatology life quality index (CDLQI), 1995-2012. Br J Dermatol. 2013;169(4):734-59.

7. Schram ME, Spuls PI, Leeflang MM, et al. EASI, (objective) SCORAD and POEM for atopic eczema: responsiveness and minimal clinically important difference. Allergy. 2012;67(1):99-106.

8. Basra MK, Salek MS, Camilleri L, et al. Determining the minimal clinically important difference and responsiveness of the dermatology life quality index (DLQI): further data. Dermatology. 2015;230(1):27-33.

9. Simpson EL, Paller AS, Siegfried EC, et al. Dupilumab efficacy and safety in adolescents with moderate-to-severe atopic dermatitis: results from a multicenter, randomized, placebo-controlled, double-blind, parallel-group, phase 3 study. In: Poster presented at the 27th European Academy of Dermatology and Venereology congress; Paris, France; September 12-16, 2018.

10. US Food and Drug Administration. (2009) Guidance for industry. Patient-reported outcome measures: use in medical product development to support labeling claims. https://www.fda.gov/downloads/ drugs/guidances/ucm193282.pdf. Accessed April 2019. 
11. Gaunt DM, Metcalfe C, Ridd M. The patient-oriented eczema measure in young children: responsiveness and minimal clinically important difference. Allergy. 2016;71(11):1620-5.
12. Howells L, Ratib S, Chalmers JR, et al. How should minimally important change scores for the patientoriented eczema measure be interpreted? A validation using varied methods. $\mathrm{Br} \mathrm{J}$ Dermatol. 2018;178(5):1135-42. 